\title{
Risk factors for small bowel obstruction after open rectal cancer resection
}

\author{
Kevin Afshari* ${ }^{*}$, Abbas Chabok, Kenneth Smedh and Maziar Nikberg
}

\begin{abstract}
Background: Small bowel obstruction (SBO) is observed in around 10\% of patients with prior open abdominal surgery. Rectal resection causes the highest readmission rates. The aim of this study was to investigate risk factors for readmission for SBO and causes for SBO in patients who needed surgery following rectal cancer surgery.

Methods: A population-based registry with prospectively gathered data on 752 consecutive patients with rectal cancer who underwent open pelvic surgery between January 1996 and January 2017 was used. Univariable and multivariable regression analysis was performed, and the risk of SBO was assessed.

Results: In total, 84 patients (11\%) developed SBO after a median follow-up time of 48 months. Of these patients, $57 \%$ developed SBO during the 1st year after rectal cancer surgery. Surgery for SBO was performed in 32 patients (4.3\%), and the cause of SBO was stoma-related in one-fourth of these patients. In the univariable analysis previous RT and re-laparotomy were found as risk factors for readmission for SBO. Re-laparotomy was an independent risk factor for readmission for SBO (OR 2.824, $\mathrm{Cl} 1.129-7.065, P=0.026)$ in the multivariable analysis, but not for surgery for SBO. Rectal resection without anastomoses, splenic flexors mobilization, intraoperative bleeding, operative time were not found as risk factors for SBO.

Conclusions: One-tenth of rectal cancer patients who had open surgery developed SBO, most commonly within the 1st postoperative year. The risk of SBO is greatest in patients with complications after rectal cancer resection that result in a re-laparotomy.
\end{abstract}

Keywords: Small bowel obstruction, Rectal cancer, Surgery, Admission, Risk factors

\section{Background}

Abdominal surgical trauma causes adhesion in almost all patients [1]. Although the majority of patients are asymptomatic, a significant number suffer from small bowel obstruction (SBO) [2], female infertility [3], and chronic pain [4]. After abdominal surgery, SBO has been observed in $9 \%$ of patients [5]. A readmission rate of $30 \%$ during a 10-year period [6] has been reported, and most cases occur within in the 1st year after abdominal surgery

*Correspondence: kevin.afshari@regionvastmanland.se

Colorectal Unit, Department of Surgery and Centre for Clinical Research

of Uppsala University, Västmanland's Hospital Västerås, 72189 Västerås, Sweden
[7]. Rectal cancer surgery causes the highest readmission rates $[5,8,9]$.

Several studies have focused on outcomes in adhesionrelated SBO. Mortality rates in SBO surgery are reported to be up to $10 \%$ [10], rising to $15 \%$ [11], when small bowel resection is performed, with a $33 \%$ risk of inadvertent enterotomy during surgery for bowel obstruction [12].

Surgery for rectal cancer has the highest adhesionrelated readmissions; therefore, it is important to elucidate the risk factors for SBO related to rectal cancer surgery. Some surgeons believe that omentoplasty to fill the pelvis lowers the risk of SBO. An empty pelvis after abdominoperineal excision (APE) or Hartmann's procedure (HP) may also lead to a higher risk of SBO compared 
with anterior resection (AR). However, results from studies are not consistent [13].

There are few studies on rectal cancer and many studies are retrospective, using ICD-10 codes to identify patients and focus only on adhesion-related SBO. The aim of the present study was to investigate risk factors for SBO readmission and the different causes of SBO in patients with prior open rectal cancer surgery, in a cohort where data has been collected prospectively.

\section{Methods}

This study was based on prospectively collected data from a local population-based registry on all openly operated patients with rectal cancer diagnosed between January 1996 and January 2017 in Västmanland county. This comprehensive local data set includes detailed pre-, peri-, and postoperative data, as well as follow-up data with information on admission for SBO with or without surgery. Patients were scheduled for follow-up at 1, 6, 12, $24,36,48$, and 60 months after surgery.

Rectal cancer was identified with a rigid rectoscope and defined as an adenocarcinoma with a distal margin within $15 \mathrm{~cm}$ from the anal verge.

\section{Study population}

In total, 1136 patients were diagnosed with rectal cancer during this period. Of these, 159 did not undergo any intervention, 191 underwent interventions such as endoscopic resection, polypectomy, stoma formation, or stent placement and 34 underwent laparoscopic surgery (starting from 2015). In total, 752 underwent open rectal tumor resection who were included in the study.

\section{Data variables}

All data were collected prospectively at each follow-up. In patients with SBO during the follow-up period, additional data were retrieved from medical records, and supplementary information was gathered from the surgical platform to identify those patients who had been admitted and treated without or with surgery for SBO. For all patients who underwent surgery for their SBO the cause of obstruction was registered after surgical notes were scrutinized.

In 60 patients, follow-up data were collected retrospectively from their medical records because of missing data in the registry. Patients were noted as lost to follow-up if they had died or moved outside the county. In the case of death, data were collected through the Swedish Death Registry.

Preoperative screening for metastases was routinely performed. Up until 2002, chest radiography and liver ultrasonography were used, and thereafter, computed tomography of the thorax and abdomen. Magnetic resonance imaging of the pelvis was used for local staging in almost all cases. Stage was defined according to the 6th American Joint Committee of Cancer (AJCC) TNM classification.

\section{Definitions}

SBO was defined as any admission to the hospital or surgery, due to small bowel obstruction occurring after 30 days postoperatively, diagnosed with radiography. Early post-operative bowel paralysis was not registered as SBO but rather as a post-operative complication.

Patients with clinical signs of SBO (abdominal pain, vomiting, distention) were diagnosed on plain abdominal X-ray or computed tomography $(\mathrm{CT})$. Conservative treatment involved nasogastric tube, intravenous fluids for resuscitation and serial X-rays for diagnostic and therapeutic purposes. In patients with contrast passage to the colon at the abdominal radiography, the nasogastric tube was removed. If the contrast failed to reach the colon or if the patient's condition deteriorated, patients underwent surgery. Surgical treatment involved exploratory laparotomy with adhesiolysis, with or without small bowel resection.

The scoring system of the American Society of Anesthesiologists (ASA) was used to determine a patient's degree of comorbidity, and performance status was graded according to World Health Organization performance status (WHO).

Based on the type of surgery, two groups where created: resection with and without anastomosis. Resection with anastomosis included AR and patients who had procto-colectomy with pelvic reservoir. The group without anastomosis included HP and APE.

Surgical details of the rectal cancer surgery have been described previously $[14,15]$. The omentum majus was placed along with the descending colon, but no omentoplasty was performed to fill the pelvis in any patient. Adhesion preventive solutions were not used. Resection of other organs was defined as an en bloc resection of adjacent organs in locally advanced rectal tumors. This cohort included all resected rectal tumors both curatively intended and palliatively resected. Postoperative surgical complications within 30 days were anastomotic leakage, infected hematoma, pelvic abscess, colovaginal fistula, bleeding, abdominal wall dehiscence, and stoma complications. Other risk factors of interest were the amount of surgical trauma, including minimal invasive surgery, mobilization of splenic flexure, and re-laparotomy due to complications. Incisional hernia was observed either through a clinical examination during follow-up or using computer tomography. Radiotherapy (RT) included both patients who had undergone RT because of rectal cancer or because of other previously treated pelvic tumors. 


\section{Statistical analysis}

Continuous data were reported as mean \pm standard deviation (SD) or median with range. Categorical data were analyzed for differences in proportions using the $x^{2}$-test or Fisher's exact test for low numbers. Univariable and multivariable analyses for factors affecting admission or surgery for SBO were performed using binary logistic regression. The multivariable analyses used all possible factors affecting SBO admission. For incisional hernia, Spearman correlation was used. $P$ values $<0.05$ were considered statistically significant. Data were analyzed using SPSS software (v. 24; IBM Corp., Armonk, NY, USA).

\section{Results}

A total of 752 patients underwent open rectal cancer resection between 1996 and 2017 and were included in the study. Of those, 733 patient (97.5\%) were followed up for more than 6 months, and 19 patients (2.5\%) died before the 6-month follow-up. The median follow-up time was 48 months (range: 0-96 months).

The patients' characteristics are summarized in Table 1. The mean age was $68 \pm 10$ years. Sixty-two percent were male, and the majority were ASA $1-2$. Most patients had a WHO performance status of $0-1$. Sixty-two percent of the patients underwent AR, and the splenic flexure was mobilized in $60 \%$. In total, 579 patients $(77 \%)$ had RT, whereof 18 patients $(2.4 \%)$ had received RT previously for prostatic or gynecological cancer.

Surgical complications were seen in 158 patients (21\%) (Table 2) and in 28 patients the surgical complications resulted in a re-laparotomy. Re-laparotomy due to complications were associated with an increased risk of admission for SBO both in the univariable (OR 2.853, CI 1.174-6.936, $\mathrm{P}=0.021$ ) and multivariable analysis (OR 2.824, CI 1.129-7.065, $\mathrm{P}=0.026$ ) (Table 3). Previous RT was associated with increased risk of hospital admissions for SBO in the univariable analysis (OR 2.112, CI 1.0934.078, $\mathrm{P}=0.026$ ) but not in the multivariable analysis (Table 3).

SBO was diagnosed in 84 patients (11\%), and in 48 patients (57\%), SBO occurred during the 1st postoperative year. Surgery for SBO was performed in 32 patients $(4.3 \%)$ admitted during the study period. The 30-day mortality after surgery for SBO was $3 \%(N=1)$, and the 90-day mortality was $9 \%(N=3)$.

In 24 patients of the 32 patients (75\%) who had surgery for SBO, adhesions were observed in the abdominal wall $(N=10)$, in the pelvis $(N=7)$, and in both the abdominal wall and pelvis $(N=7)$. The cause of SBO was in addition to adhesions, related to the stoma in 9 patients $(28 \%)$; stenosis before closure of the loop ileostomy $(N=2)$, strangulation of the small bowel around the loop ileostomy
Table 1 Demographics, patient, and surgical characteristics of patients undergoing surgery for rectal cancer in Västmanland county between 1996 and 2017

\begin{tabular}{|c|c|c|c|}
\hline Parameters & $\begin{array}{l}\text { Total cohort } \\
N=752\end{array}$ & $\begin{array}{l}\text { With SBO } \\
N=84\end{array}$ & $\begin{array}{l}\text { Without SBO } \\
N=668\end{array}$ \\
\hline Age $\left(\right.$ years) ${ }^{b}$ & $69(32-88)$ & $66(42-87)$ & $70(32-88)$ \\
\hline Gender M: F & 469: 283(62:38) & $53: 31(63: 37)$ & $416: 252(62: 38)$ \\
\hline Body mass index $\left(\mathrm{kg} / \mathrm{m}^{2}\right)^{\mathrm{c}}$ & $26 \pm 4$ & $25 \pm 4$ & $25 \pm 4$ \\
\hline Missing & 5 & 0 & 5 \\
\hline \multicolumn{4}{|l|}{ ASA score } \\
\hline$|-| \mid$ & $524(70)$ & $61(73)$ & $463(69)$ \\
\hline III-IV & $228(30)$ & $23(27)$ & $205(31)$ \\
\hline \multicolumn{4}{|l|}{ WHO PS } \\
\hline I & $435(58)$ & $51(61)$ & $384(57)$ \\
\hline$\|$ & $282(37)$ & $30(36)$ & $252(38)$ \\
\hline III & $28(4)$ & $3(3)$ & $25(4)$ \\
\hline IV & $6(1)$ & $0(0)$ & $6(1)$ \\
\hline \multicolumn{4}{|l|}{ TNM stage } \\
\hline$|-| \mid$ & $404(54)$ & $42(50)$ & $362(54)$ \\
\hline III & $256(34)$ & $29(35)$ & $227(34)$ \\
\hline IV & $91(12)$ & $13(15)$ & $78(12)$ \\
\hline $\begin{array}{l}\text { Preoperative } \\
\text { radiotherapy }{ }^{\mathrm{a}}\end{array}$ & $579(77)$ & $73(87)$ & $506(76)$ \\
\hline Missing & 1 & 0 & 1 \\
\hline \multicolumn{4}{|l|}{ Type of surgery } \\
\hline Anterior resection & $468(62)$ & $48(57)$ & $420(63)$ \\
\hline Hartmann's procedure & $84(11)$ & $7(8)$ & $77(11)$ \\
\hline $\begin{array}{l}\text { Abdominoperineal } \\
\text { excision }\end{array}$ & $184(25)$ & $27(32)$ & $157(24)$ \\
\hline Proctocolectomy & $16(2)$ & $2(3)$ & $14(2)$ \\
\hline $\begin{array}{l}\text { Mobilization of splenic } \\
\text { flexure }\end{array}$ & $454(60)$ & $51(61)$ & $403(60)$ \\
\hline Resection of other organs & $202(27)$ & $25(30)$ & $177(27)$ \\
\hline $\begin{array}{l}\text { Intraoperative bleeding } \\
(\mathrm{mL})^{c}\end{array}$ & $689 \pm 618$ & $723 \pm 604$ & $685 \pm 620$ \\
\hline Missing & 3 & 0 & 3 \\
\hline Operative time $(\mathrm{min})^{c}$ & $293 \pm 76$ & $302 \pm 70$ & $292 \pm 76$ \\
\hline Missing & 5 & 0 & 5 \\
\hline Re-laparotomy & $28(4)$ & $7(8)$ & $21(3)$ \\
\hline Incisional hernia & $69(9)$ & $10(12)$ & $59(9)$ \\
\hline
\end{tabular}

Other values in parentheses are percentages

SBO Small bowel obstruction, ASA American Society of Anesthesiologists, WHO $P S$ World Health Organization performance status

a Preoperative radiotherapy is any radiotherapy given at any time prior to surgery for rectal cancer, including radiotherapy for cancers other than rectal cancer

b Values are median (range)

c Continuous values are presented as mean \pm standard deviation

$(N=3)$, and stenoses after closure $(N=4)$. The remaining causes of SBO were abdominal malignancy $(N=4)$, such as carcinomatosis or recurrent tumor, and unspecified in two. 
Table 2 Total number of complications after rectal cancer surgery in Västmanland county between 1996 and 2017 and complications leading to re-laparotomy

\begin{tabular}{lcc}
\hline Complications & $\mathbf{N = 1 5 8}$ & $\begin{array}{l}\text { Re-laparotomy } \\
(\mathbf{N}=\mathbf{2 8})\end{array}$ \\
\hline Anastomotic leakage & $31(20)$ & 10 \\
Abscess in the lower abdomen & $10(6)$ & 1 \\
Bleeding & $1(1)$ & 1 \\
Abdominal wall dehiscence & $3(2)$ & 3 \\
Stomal complications & $10(6)$ & 3 \\
Intestinal injury & $4(2)$ & 4 \\
lleus & $9(6)$ & 4 \\
Wound infection & $46(29)$ & \\
Perineal wound infection & $42(27)$ & \\
Colovaginal fistula & $2(1)$ & \\
Other & & $2^{\mathrm{a}}$
\end{tabular}

Values in parentheses are percentages

a Two patient had normal finding at laparotomy

Morbidity, age, type of rectal cancer surgery (i.e., with or without anastomosis), more extensive surgical dissection planes, such as mobilization of the left flexure and resection of other organs, were not associated with the rate of hospital admissions (Table 3). Of those patients who underwent an AR, 74\% received a loop ileostomy with no increased risk of admission due to SBO ( $P=0.569$, data not shown). None of the analyzed factors resulted in an increased risk of surgical outcome for SBO in the logistic regression analysis (data not shown). Sixtynine patients developed incisional hernia, and no correlation was found between admission and surgery for SBO and incisional hernia (data not shown).

\section{Discussion}

The long-term risk of SBO after rectal cancer surgery was $11 \%$, and most patients were admitted in the 1st postoperative year. Surgery for SBO was performed in $4.3 \%$ of patients, and the causes were related to adhesions in $75 \%$ and some in addition had SBO related to their stoma. Patients who had previous RT had a twofold increased risk of SBO necessitating hospital admission and patients with postoperative complications that resulted in relaparotomy had a threefold increased risk for future admission due to SBO. However, the type of rectal cancer surgery (i.e., with or without anastomosis) or more extensive surgical dissection were not associated with hospital admission rates or surgery for SBO.

We present one of the largest prospectively collected cohorts on rectal cancer patients who had open surgery and the risk of admission due to SBO. Our data are in accordance with a previously reported study based on a Swedish inpatient registry [9]. The risk of surgery for SBO has been studied extensively. One study based on data from a Danish registry found this risk to be $4.5 \%$ [16], which is the same as that in the present study. Another study found the risk to be between 19 and 66\% [17]. In addition, the increased risk of SBO during the 1st year after surgery $(57 \%)$ is in concordance with previous studies that report risks of $22-60 \%[6,7,18]$.

Within the cohort, we report a high rate of loop ileostomy-related SBO with various causes both before and after closure, which together with other negative ileostomy-related outcomes, such as poorer functional outcomes and costs, raises concern about the routine use of loop ileostomy [19, 20].

Stoma formation is a risk factor for SBO in colorectal surgery, but no consensus has been reached regarding whether the type of stoma affects the SBO outcome. One study found an increased risk of SBO in colorectal surgery in those with stoma, but no difference in the incidence of SBO was found between ileostomy or colostomy [21].

Gastrointestinal dysfunction, especially bowel obstruction, is well known after RT $[22,23]$. In the Swedish Rectal Cancer Trial [22], an approximate twofold increased risk of bowel obstruction was shown as both an early and late adverse effect of RT. This was also found in this study in the univariable analyses for admission due to $\mathrm{SBO}$ however not in the multivariable analysis and not for surgery for SBO. This is probably because of different study periods, differences in radiation fields or due to type II error.

Interestingly, the type of rectal cancer surgery with regard to resection with and without anastomosis or the extent of dissection was not a risk factor for admission and surgery for SBO. However, surgical complications that resulted in re-laparotomy after rectal cancer surgery were an independent risk factor for admission for $\mathrm{SBO}$. One can speculate that this is probably partly due to peritoneal inflammation related to the complications, and partly to secondary surgical trauma, which, in turn, causes additional adhesions.

To reduce the risk of ileus, omentoplasty is performed by some surgeons to prevent the decent of the small bowel in the empty pelvic cavity after APE and HP, even though, to our knowledge, without any evidence of reducing this risk. On the contrary, a recent study reported that the readmission and reintervention rates for SBO did not differ, with or without omentoplasty [13]. In the present cohort, none of the patients had an omentoplasty, and we could not find any difference regarding SBO admissions between those with anastomosis, i.e., filled pelvis after AR, versus those without an anastomosis, i.e., after APE and HP. 
Table 3 Univariable and multivariable logistic regression analysis of patients undergoing surgery for rectal cancer in Västmanland county between 1996 and 2017 who were admitted for small bowel obstruction (SBO)

\begin{tabular}{|c|c|c|c|c|c|c|}
\hline \multirow[t]{2}{*}{ Admission for SBO } & \multicolumn{3}{|c|}{ Univariable } & \multicolumn{3}{|c|}{ Multivariable } \\
\hline & OR & $95 \% \mathrm{Cl}$ & $P$ & OR & $95 \% \mathrm{Cl}$ & $P$ \\
\hline Age & 0.981 & $0.961-1.002$ & 0.082 & 0.981 & $0.958-1.005$ & 0.113 \\
\hline \multicolumn{7}{|l|}{ Gender } \\
\hline Female & 1 & & 0.884 & 1 & & 0.941 \\
\hline Male & 1.036 & $0.647-1.657$ & & 1.019 & $0.616-1.688$ & \\
\hline Body mass index & 0.975 & $0.920-1.034$ & 0.404 & 0.969 & $0.910-1.031$ & 0.321 \\
\hline \multicolumn{7}{|l|}{ ASA score } \\
\hline$|-| \mid$ & 1 & & 0.535 & 1 & & 0.762 \\
\hline III-IV & 0.852 & $0.513-1.414$ & & 0.913 & $0.505-1.648$ & \\
\hline \multicolumn{7}{|l|}{ TNM stage } \\
\hline$|-| \mid$ & 1 & & & 1 & & \\
\hline III & 1.101 & $0.667-1.818$ & 0.707 & 1.148 & $0.682-1.932$ & 0.603 \\
\hline IV & 1.437 & $0.736-2.803$ & 0.288 & 1.346 & $0.660-2.743$ & 0.414 \\
\hline \multicolumn{7}{|l|}{ Preoperative radiotherapy ${ }^{\mathrm{a}}$} \\
\hline No & 1 & & 0.026 & 1 & & 0.071 \\
\hline Yes & 2.112 & $1.093-4.078$ & & 1.932 & $0.945-3.951$ & \\
\hline \multicolumn{7}{|l|}{ Type of surgery } \\
\hline Resection with anastomosis & 1 & & 0.321 & 1 & & 0.225 \\
\hline Resection without anastomosis & 1.262 & $0.797-1.999$ & & 1.614 & $0.745-3.495$ & \\
\hline \multicolumn{7}{|l|}{ Mobilization of splenic flexure } \\
\hline No & 1 & & 0.946 & 1 & & 0.271 \\
\hline Yes & 1.016 & $0.639-1.617$ & & 1.519 & $0.722-3.197$ & \\
\hline \multicolumn{7}{|l|}{ Resection of other organs } \\
\hline No & 1 & & 0.525 & 1 & & 0.832 \\
\hline Yes & 1.175 & $0.714-1.935$ & & 0.938 & $0.519-1.696$ & \\
\hline Intraoperative bleeding & 1 & $1.000-1.000$ & 0.596 & 1 & $1.000-1.000$ & 0.853 \\
\hline Operative time & 1.002 & $0.999-1.005$ & 0.232 & 1 & $0.996-1.004$ & 0.865 \\
\hline \multicolumn{7}{|l|}{ Re-laparotomy } \\
\hline No & 1 & & 0.021 & 1 & & 0.026 \\
\hline Yes & 2.853 & $1.174-6.936$ & & 2.824 & $1.129-7.065$ & \\
\hline
\end{tabular}

ASA American Society of Anesthesiologists, $\mathrm{Cl}$ confidence interval, OR odds ratio

a Preoperative radiotherapy is any radiotherapy given at any time prior to surgery for rectal cancer, including radiotherapy for cancers other than rectal cancer

The main strength of this study is its population-based design with prospectively registered data, which limits the bias associated with retrospective studies. The cohort was homogenous, with only rectal cancer patients operated with the intent of local radical resection. As this was a single center study, the surgery was standardized, and only few colorectal surgeons operated on the patients. All variables were registered at each follow-up visit by the same surgeons, and consensus was reached on the definitions of all variables. For patients with SBO identified in the registry, medical records were scrutinized and etiologies behind $\mathrm{SBO}$ were identified.

The results should though be interpreted cautiously because of the low number of patients with SBO. In the present study, we included patients regardless of previous surgery before the index surgery, which may have introduced bias.

\section{Conclusion}

One-tenth of rectal cancer patients who had open surgery developed SBO during follow-up, most commonly within the 1st postoperative year. The risk of SBO is greatest in patients with complications after rectal cancer resection that result in a re-laparotomy. The type of resection surgery, with or without anastomosis, was not a risk factor for SBO. 


\section{Abbreviations}

AJCC: American Joint Committee of Cancer; ASA: American Society of Anesthesiologist; APE: Abdominoperineal excision; AR: Anterior resection; HP: Hartmann's procedure; SBO: Small bowel obstruction; WHO: World Health Organization; RT: Radiotherapy.

\section{Acknowledgements}

Special thanks to Malin Engdahl for her contributions.

\section{Authors' contributions}

Guarantor of the integrity of the study: MN, KS, AC, KA. Study concept: MN. Study design: MN, KS. Definition of intellectual content: MN, KS, AC, KA. Literature research: KA. Data acquisition: MN, KS, AC, KA. Data analysis: MN, KS, AC, KA. Statistical analysis: MN, KA. Manuscript preparation: MN, KS, AC, KA. Manuscript editing: MN, KS, AC, KA. Manuscript review: MN, KS, AC, KA. All authors read and approved the final manuscript.

\section{Funding}

Open Access funding provided by Uppsala University.

\section{Availability of data and materials}

The datasets used and/or analysed during the current study are available from the corresponding author on reasonable request.

\section{Ethics approval and consent to participate}

Written and oral informed consent is received by all participants. The study was approved by the Regional Ethics Review Board in Uppsala and complied with the Declaration of Helsinki (Dnr 2017/353).

\section{Consent for publication}

Written and oral informed consent is received by all participants for publication.

\section{Competing interests}

The authors declare that they have no competing interests.

Received: 13 August 2020 Accepted: 20 January 2021

Published online: 28 January 2021

\section{References}

1. Menzies D, Ellis H. Intestinal obstruction from adhesions-how big is the problem? Ann R Coll Surg Engl. 1990;72(1):60-3.

2. Menzies D. Postoperative adhesions: their treatment and relevance in clinical practice. Ann R Coll Surg Engl. 1993;75(3):147-53.

3. Hershlag A, Diamond MP, DeCherney AH. Adhesiolysis. Clin Obstet Gynecol. 1991:34(2):395-402.

4. Rapkin AJ. Adhesions and pelvic pain: a retrospective study. Obstet Gynecol. 1986;68(1):13-5.

5. ten Broek RP, Issa Y, van Santbrink EJ, Bouvy ND, Kruitwagen RF, Jeekel J, et al. Burden of adhesions in abdominal and pelvic surgery: systematic review and met-analysis. BMJ. 2013;347:f5588.

6. Parker MC, Ellis H, Moran BJ, Thompson JN, Wilson MS, Menzies D, et al. Postoperative adhesions: ten-year follow-up of 12,584 patients undergoing lower abdominal surgery. Dis Colon Rectum. 2001;44(6):822-9 (discussion 9-30).

7. Ellis H, Moran BJ, Thompson JN, Parker MC, Wilson MS, Menzies D, et al. Adhesion-related hospital readmissions after abdominal and pelvic surgery: a retrospective cohort study. Lancet. 1999;353(9163):1476-80.
8. Parker MC, Wilson MS, Menzies D, Sunderland G, Clark DN, Knight AD, et al. The SCAR-3 study: 5-year adhesion-related readmission risk following lower abdominal surgical procedures. Colorectal Dis. 2005;7(6):551-8.

9. Angenete E, Jacobsson A, Gellerstedt M, Haglind E. Effect of laparoscopy on the risk of small-bowel obstruction: a population-based register study. Arch Surg. 2012;147(4):359-65.

10. Menzies D, Parker M, Hoare R, Knight A. Small bowel obstruction due to postoperative adhesions: treatment patterns and associated costs in 110 hospital admissions. Ann R Coll Surg Engl. 2001;83(1):40-6.

11. Wysocki A, Pozniczek M, Kulawik J, Krzywon J. Peritoneal adhesions as cause of small bowel obstruction. Przegl Lek. 2003;60(Suppl 7):32-5.

12. Van Der Krabben AA, Dijkstra FR, Nieuwenhuijzen M, Reijnen MM Schaapveld M, Van Goor H. Morbidity and mortality of inadvertent enterotomy during adhesiotomy. Br J Surg. 2000;87(4):467-71.

13. Blok RD, Musters GD, Borstlap WAA, Buskens CJ, Bemelman WA, Tanis PJ, et al. Snapshot study on the value of omentoplasty in abdominoperineal resection with primary perineal closure for rectal cancer. Ann Surg Oncol. 2018;25(3):729-36

14. Nikberg M, Kindler C, Chabok A, Letocha H, Shetye J, Smedh K. Circumferential resection margin as a prognostic marker in the modern multidisciplinary management of rectal cancer. Dis Colon Rectum. 2015;58(3):275-82.

15. Khani MH, Smedh K, Kraaz W. Is the circumferential resection margin a predictor of local recurrence after preoperative radiotherapy and optimal surgery for rectal carcinoma? Colorectal Dis. 2007;9(8):706-12.

16. Andersen $P$, Jensen KK, Erichsen R, Froslev T, Krarup PM, Madsen MR, et al. Nationwide population-based cohort study to assess risk of surgery for adhesive small bowel obstruction following open or laparoscopic rectal cancer resection. BJS Open. 2017;1(2):30-8.

17. Yang KM, Yu CS, Lee JL, Kim CW, Yoon YS, Park IJ, et al. The long-term outcomes of recurrent adhesive small bowel obstruction after colorectal cancer surgery favor surgical management. Medicine (Baltimore). 2017:96(43):e8316.

18. Edna TH, Bjerkeset T. Small bowel obstruction in patients previously operated on for colorectal cancer. Eur J Surg. 1998;164(8):587-92.

19. Gadan S, Floodeen H, Lindgren R, Matthiessen P. Does a defunctioning stoma impair anorectal function after low anterior resection of the rectum for cancer? A 12-year follow-up of a randomized multicenter trial. Dis Colon Rectum. 2017;60(8):800-6.

20. Park J, Angenete E, Bock D, Correa-Marinez A, Danielsen AK, Gehrman J, et al. Cost analysis in a randomized trial of early closure of a temporary ileostomy after rectal resection for cancer (EASY trial). Surg Endosc. 2019;34:69-76.

21. Smolarek S, Shalaby M, Paolo Angelucci G, Missori G, Capuano I, Franceschilli L, et al. Small-bowel obstruction secondary to adhesions after open or laparoscopic colorectal surgery. JSLS. 2016;20(4).

22. Birgisson H, Pahlman L, Gunnarsson U, Glimelius B, Swedish Rectal Cancer Trial G. Adverse effects of preoperative radiation therapy for rectal cancer: long-term follow-up of the Swedish Rectal Cancer Trial. J Clin Oncol. 2005;23(34):8697-705.

23. Peeters KC, van de Velde CJ, Leer JW, Martijn H, Junggeburt JM, Kranenbarg EK, et al. Late side effects of short-course preoperative radiotherapy combined with total mesorectal excision for rectal cancer: increased bowel dysfunction in irradiated patients - a Dutch colorectal cancer group study. J Clin Oncol. 2005;23(25):6199-206.

\section{Publisher's Note}

Springer Nature remains neutral with regard to jurisdictional claims in published maps and institutional affiliations. 